\title{
Wie unnütz ist das Wissen von Bouvard und Pécuchet?
}

Bouvard et Pécuchet ist Gustave Flauberts letzter Roman. ${ }^{1}$ Er blieb unvollendet und erschien, nachdem Flaubert im Mai 1880 mit 58 Jahren gestorben war, zunächst in Fortsetzungen als Zeitschriftenpublikation, dann im März 1881 als Buch. Die überaus spärliche Handlung scheint fast ausschließlich ein ehikel zur Darstellung von Wissen zu sein; allerdings auf merkwürdige Weise. Bouvard und Pécuchet, zwei Pariser Angestellte - Schreiber, "copistes" (48) -, ziehen sich mit einem kleinen ererbten Vermögen in das fiktive normannische Provinzstädtchen Chavignolles zurück, wo sie sich über etliche Jahre mit praktischen und theoretischen Wissenschaften befassen, von der Agrikultur über die Naturwissenschaften und Medizin, Geschichte, Literatur und Politik, bis hin zum Spiritismus, der Religion und der Pädagogik. Ihr immer neuer dilettantischer Enthusiasmus endet jedes Mal in Enttäuschung oder Langeweile, bis sie sich wieder auf das beschränken, was sie am besten (oder als Einziges) können - abschreiben. So stellt sich gemäß Flauberts nachgelassenen Notizen ein möglicher Abschluss des im Manuskript abbrechenden zehnten Kapitels dar: "Bonne idée nourrie en secret par chacun d'eux. Ils se la dissimulent [...] puis se la communiquent simultanément: copier. [...] Ils s'y mettent.« $(399)^{2}$

Während man mit Blick auf die Romanhandlung zunächst von völlig nutzlosem Wissen sprechen möchte, zeigt die Betonung der Schreibszene, dass es mit jenem oberflächlichen Befund nicht sein Bewenden haben kann. Vielmehr liegt hier ein komplexes Spannungsverhältnis von Wissen und Schreiben vor, das bei einem poetologisch so reflektierten Autor wie Flaubert nicht ohne Auswirkungen auf das Verhältnis von Wissen und Literatur

1 Zitiert wird nach der Ausgabe Gustave Flaubert, Bouvard et Pécuchet. Avec des fragments du "second volume", dont le Dictionnaire des idées reçues, hg. von Stéphanie Dord-Crouslé, Paris 1999. Deutsche Zitate nach der Ausgabe: Gustave Flaubert, Bouvard und Pécuchet, übers. von Thomas Dobberkau, Berlin 1980. Zitate aus beiden Ausgaben werden im Folgenden nur mit Angabe der Seitenzahl nachgewiesen (die deutschen zumeist in den Fußnoten).

2 "Ein guter Gedanke, den beide heimlich hegen. Sie verschweigen ihn voreinander [...] dann endlich teilen sie ihn sich gleichzeitig mit: Abschreiben wie früher [...]. Sie gehen ans Werk." (347) Die Übersetzung folgt der Pléiade-Ausgabe (Paris 1975, S. 301), in der die Formel lautet »copier, comme autrefois«. 
sein kann. Auch unabhängig davon lässt sich die Frage nach dem Nutzen des Wissens nicht rein utilitaristisch formulieren und beantworten. Zum Beleg sei auf die semantische Auffächerung von snutzen und snützlich im Französischen hingewiesen, das in diesem Fall ja immer mit zu befragen ist. 'Nützlich kann utile sein, aber auch bénéfque oder productif; snutzen heißt être utile, aber auch servir; sunnütz könnte man außer mit inutile auch mit vain übersetzen; hinzu käme die Frageformel à quoi bon ?, entsprechend der lateinischen Wendung cui bono?, in der snutzen als sgut sein für erscheint. Diese Anreicherung im Sprachgebrauch zeigt, dass mit der Frage nach dem Nutzen des Wissens ein grammatisch-logisch weitläufiger Bereich betreten ist, in dem sich die Finalität des >um zu< und `damit mit der Konsekutivität des sso dass` überschneidet, und dass diese Überschneidung noch dazu die wissens-ethischen Probleme des >dienen zu< und 'gut sein für aufwirft.

Was also bewirkt, wohin führt Wissen in Bouvard et Pécuchet, wozu dient es, wofür ist es gut? Diese Frage fordert zunächst eine Vorbemerkung zum Problem des Enzyklopädischen in seinem Zusammenhang mit dem Nutzen des Wissens in der und für die Literatur. Im Anschluss daran werde ich für Flauberts Roman drei Ebenen unterscheiden: 1. die Ebene der fiktiven Romanhandlung, auf der sich das Wissen der beiden Protagonisten vollzieht, 2. die Ebene der Textgenese, auf der Wissen als materielle Grundlage des Romans fungiert, 3. die Ebene der Poetologie, auf der sich Flauberts Romankunst als das (angestrebte) Andere des Wissens profiliert.

\section{Enzyklopädie und Roman}

Flaubert hat Bouvard et Pécuchet wiederholt mit dem schlechthin maximalen Ordnungsmodell des Wissens in Verbindung gesetzt: mit dem Modell der Enzyklopädie. ${ }^{3}$ In brieflichen Äußerungen während der jahrelangen Arbeit

3 In der neueren Flaubert-Forschung ist der Enzyklopädie-Hinweis topisch: »ce roman encyclopédique« (Atsushi Yamazaki, Bouvard et Pécuchet ou la pulvérisation de la philosophie, in: Études de Langue et Littérature Françaises 90 (2007), S. 81-100, hier S. 81), "enzyklopädische[r] Roman des Wissens" (Moritz Baßler, Erhabene und komische Kopisten. Roland Barthes über Gustave Flauberts »Bouvard und Pécuchet«, in: Heinz-Peter Preußer/Matthias Wilde (Hg.), Kulturphilosophen als Leser. Porträts literarischer Lektüren, Göttingen 2006, S. 315-321, hier S. 321). In der wissensgeschichtlich vergleichenden Goethe-Melville-Flaubert-Analyse von Claire Barel-Moisan u.a. figuriert das Enzyklopädische unter den geradezu lehrbuchmäßig abzuschreitenden Themen (Fictions du savoir, savoirs de la fiction. Goethe, "Les affinités électives", Melville, "Mardi«, Flaubert, 
am Roman nannte er das entstehende Werk eine Enzyklopädie der Dummheit, sei es der modernen oder der allgemeinen menschlichen, ${ }^{4}$ und sprach von einer »espèce d'encyclopédie critique en farce«. ${ }^{5}$ Diese oft zitierte, bündige Formel fasst die spezifische Art (espèce) des Romans im Ineinander von Enzyklopädie, Kritik und Farce: eine kritische Enzyklopädie rals oder sin Gestalt einer Farce - also eines probaten literarischen Mittels, wenn es um die Erniedrigung des Hohen, Erhabenen geht. ${ }^{6}$ Demnach wäre die Farce in Flauberts Formel als Instanz der Kritik, genauer gesagt: der kritischen Destruktion des enzyklopädischen Konzepts einer Totalisierung des Wissens zu verstehen.

Allerdings ist 'Kritik im französischen Sprachraum schon lange vor Flaubert integraler Bestandteil enzyklopädischer Unternehmungen, die die Gesamtheit des Wissens ihrer Zeit abbilden sollen. Zuerst findet sich diese Kennzeichnung in Pierre Bayles Dictionnaire historique et critique (1695-1697). Während es Bayle noch vorwiegend bei der impliziten Aufforderung an seine Leser belässt, als kritische Selbstdenker die Widersprüche in den von ihm unverbunden nebeneinandergestellten divergierenden Lehrmeinungen zu erkennen, beruht ein knappes Jahrhundert später Diderots und d'Alemberts aufklärerische Encyclopédie (1751-1780) auf Kritik als einem Schlüsselkonzept der Wissenserfassung und -darstellung. ${ }^{7}$ Kritik ist dort die Kunst-

»Bouvard et Pécuchet«, Neuilly-sur-Seine 2011, S. 204-214: "Encyclopédies et dictionnaires«, darin zu Bouvard et Pécuchet S. 209-214); vgl. auch Hildegard Haberl, Écriture encyclopédique - écriture romanesque. Représentation et critique du savoir dans le roman allemand et français de Goethe à Flaubert, Thèse, EHESS/Universität Wien 2010. Besonders differenzierte Auseinandersetzungen finden sich bei Claudine Cohen, Bouvard et Pécuchet réécrivent les sciences, in: Alliage 37/38 (1998), online unter http://www. tribunes.com/tribune/alliage/37-38/cohen.htm [Datum des letzten Zugriffs: 23.12.2015] und bei Dietrich Scholler, Umzug nach Encyclopaedia. Zur narrativen Inszenierung des Wissens in Flauberts Bouvard et Pécuchet, Berlin 2002.

4 "Ce sera une espèce d'encyclopédie de la Bêtise moderne« (Brief an Adèle Perrot, 17.10.1872, Correspondance, Bd. 4, S. 590); "L'ouvrage que je fais pourrait avoir comme sous-titrer sencyclopédie de la Bêtise humaine« (Brief an Raoul-Duval, 13.10.1879, Correspondance, Bd. 5, S. 535). Briefzitate folgen der Pléiade-Ausgabe: Gustave Flaubert, Correspondance, hg. von Jean Bruneau und Yves Leclerc, 5 Bde., Paris 1973-2007. Zum programmatischen Stellenwert der Briefe Flauberts vgl. Gerhard Walter Frey, Die ästhetische Begriffswelt Flauberts. Studien zu der ästhetischen Terminologie der Briefe Flauberts, München 1972.

5 Brief an Edma Roger des Genettes, 19.8.1872, Correspondance, Bd. 4, S. 559.

6 Zur theatergeschichtlichen Herkunft der Farce aus den Zwischenakten spätmittelalterlicher Mysterienspiele vgl. Alan E. Knight, The Late Medieval French Farce. A Study in Cultural Paradox, New Haven 1965.

7 Vgl. Andreas Kilcher, Mathesis und poiesis. Die Enzyklopädik der Literatur 1600 bis 2000, München 2003, S. 228. Kilcher weist darauf hin, dass seit Bayle speziell die Alpha- 
fertigkeit der Unterscheidung, die notwendig ist, um herauszufinden, was aus der Fülle des zu Wissenden überhaupt wissenswert ist. Das geschieht in Absetzung vom bloßen Vielwissen der frühneuzeitlichen Enzyklopädien oder >Polymathien`. Wie man dem diesbezüglichen Eintrag bei Diderot und d'Alembert entnehmen kann, ist unkritisches - unterscheidungsloses - Vielwissen geradezu der Inbegriff des Unnützen Wissens: Die »POLYMATHIE«, so heißt es, liefere nur eine wirre Anhäufung unnützer Kenntnisse (»un amas confus de connoissances inutiles«); stattdessen möge man sich seines Wissens nur insoweit bedienen, wie es für ein ganz bestimmtes Thema notwendig sei ("pour la nécessité seule du sujet que l'on traite«). ${ }^{8}$

Damit wird das Problem aufgeworfen, inwiefern enzyklopädisches Wissen überhaupt begrenzbar ist. Für die aufklärerische Encyclopédie ist die Frage nicht so klar zu beantworten, wie es der »POLYMATHIE«-Artikel verspricht. Immerhin führt aus diesem Artikel ein Verweis auf den schlechthin programmatischen Artikel Diderots: »Voyez ENCYCLOPÉDIE«. ${ }^{9}$ Und gerade die für dieses Werk so charakteristische Arbeit mit fortwährenden Verweisen aus einem Artikel in zahlreiche andere zeigt, dass die Identifikation der Begrenzung des Wissens mit seiner Nützlichkeit bzw. Nutzanwendung ("pour la nécessité seule du sujet que l'on traite") nicht leicht zu haben ist. Denn es ist die zyklische Struktur der enkyklios paideia, der sim Kreis laufenden Erziehung<, aufgrund derer fortwährend ein Bestandteil in den anderen greift. Damit bleibt die Enzyklopädie trotz der kritischen Wende im 18. Jahrhundert das literarische Muster des Vielwissens - auch und gerade im 19. Jahrhundert. Das gilt für Hegels Enzyklopädie der philosophischen Wissenschaften im Grundrisse (1830) ebenso wie für das Projekt einer Encyclopédie positiviste, wie es Auguste Comte in den 1840er-Jahren als zugleich systematische und progressive Anordnung aller wissenschaftlichen Disziplinen vorschwebte, oder für die Encyclopädie und Methodologie der philologischen Wissenschaften (1877), mit der August Boeckh die gesamten antiquarischen Kenntnisse der altertumskundlichen Wissenschafts- und Kulturgeschichte ordnen wollte.

Vor dem Hintergrund dieses epistemischen Optimismus scheint Bouvard et Pécuchet vor allem eines zu sein: ein Abgesang auf das enzyklopädistische Begehren der Erfassung und Wiedergabe alles irgend erreichbaren Wissens. Zugleich ist der Roman in seinen Techniken der Wissensverarbeitung

betisierung der Enzyklopädieeinträge das »adäquate Medium der Kritik älterer Wissenszusammenhänge« darstellt.

8 Art. Polymathie, in: Encyclopédie ou Dictionnaire raisonné des sciences, des arts et des métiers, Bd. 12 (1765), Ndr. Stuttgart/Bad Canstatt 1967, S. 944.

9 Ebd. 
und -verwaltung aber auch selbst ein unbestritten enzyklopädisches Unterfangen. Dieser Widerspruch, auf enzyklopädische Weise dennoch Distanz zur Emphase des Enzyklopädischen zu halten, prägt das Zusammengehen von Roman und Enzyklopädie schon seit der frühen Neuzeit, und auch die Farce ist hier von Beginn an mit im Spiel: Man denke an Rabelais' grotesken Roman Gargantua et Pantagruel, mit dem um 1530 das Wort 'Enzyklopädie in die europäische Literaturgeschichte eintritt, ${ }^{10}$ oder an Johann Fischarts Rabelais-Bearbeitung, die Geschichtklitterung, die in der Tradition des frühneuzeitlichen Gelehrtenromans steht und zugleich eine Parodie enzyklopädischer Wissensbestrebungen liefert. Auch für die polyhistorischen Romane des Barock, für die selbstreflexiv-wissenspoetischen Romane der Romantik oder für Émile Zolas Versuch, die Natur- und Sozialgeschichte des Second Empire in einen zwanzigbändigen Romanzyklus zu fassen, gilt, dass Romane niemals Enzyklopädien erster Ordnung sind und auch nicht zu sein versuchen, sondern das in sie eingegangene 'Ganze des Wissens irgendeiner Weise überbetonen und es so in seiner Thematisierung zugleich problematisieren. $^{11}$

Angesichts der komplexen Wechselbeziehungen von Enzyklopädie und Roman, von Kritik und Farce, stellt sich umso mehr die Frage danach, was das Wissen in Flauberts letztem Roman nützt, wozu es nütze oder nützlich ist, ob es unnütz ist - und wenn ja, in welcher Weise und in welchem Ausmaß.

\section{Arten der Wissensnutzung bei Flaubert}

\section{II.1 Romanhandlung: Das Wissen der beiden Protagonisten}

Möchte man das Unnütze des Wissens von Bouvard und Pécuchet belegen, wird man im Roman stets aufs Neue fündig. Flauberts Protagonisten verkörpern geradezu beispielhaft ein Wissen, das zu nichts führt. Unentwegt bringen sie sich nach Kräften auf den jeweils aktuellen Wissensstand, betreiben für kurze Zeit ihre Forschungen und lassen sie dann unverrichte-

10 Rabelais spricht vom "wahren Brunnen und Abgrund der Enzyklopädie«: »le vray puys et abysme de Encyclopedie«, http://fr.wikisource.org/wiki/Page:Rabelais_-_Pantagruel,_ ca_1530.djvu/89 [Datum des letzten Zugriffs: 23.12.2015].

11 Vgl. dazu, mit Beispielen vom 16. bis ins 21. Jahrhundert, die Beiträge in: Stefan Willer (Hg.), Roman als Enzyklopädie, Schwerpunkt der Zeitschrift arcadia 48 (2013), S. 259410 . 
ter Dinge liegen. So werden ihnen die Naturwissenschaften, eine nach der anderen, schnell zu kompliziert: »la loi des équivalents les embarrassa encore une fois. Ils tâchèrent de l'élucider avec la théorie des atomes, ce qui acheva de les perdre" (108); "Cependant toutes ces lectures avaient ébranlé leur cervelle« (125); »Les minéraux ne tardèrent pas à les fatiguer« (130). ${ }^{12}$ Bei geologischen Grabungen an der normannischen Steilküste werden sie von Feldhüter und Strandwächter verwarnt und verursachen einen beinahe tödlichen Erdrutsch. ${ }^{13}$ Als Archäologen füllen sie ihr Haus mit wertlosem Gerümpel und nennen das ihr >Museum‘; als Historiker wählen sie zum Forschungsgegenstand die maximal langweilige Gestalt des Herzogs von Angoulême, an dessen bedeutungsloser Biografie es schlechthin nichts zu erforschen und aus der es nichts zu erschließen gibt; als Literaten verzetteln sie sich zwischen grammatischen Detaildiskussionen und demonstrieren ihre völlige Unempfänglichkeit für Fragen der Ästhetik - und so fort.

In der Aufreihung immer neuer Unzulänglichkeiten liegt sowohl das Vergnügliche als auch das Monotone der Romanhandlung, wobei sich das eine kaum vom anderen trennen lässt. ${ }^{14}$ Allerdings sollte man angesichts des seriellen Scheiterns von Bouvards und Pécuchets Wissenserwerb nicht übersehen, wie wissbegierig die beiden sind und, allen Enttäuschungen zum Trotz, auch über viele Jahre hinweg bleiben. Ebenso bemerkenswert wie ihre Unfähigkeit ist die Unverdrossenheit, mit der sie ihren Weg durch die enkyklios paideia fortsetzen. Als sie etwa den Arzt von Chavignolles um eine Belehrung in Sachen Chemie angehen, sehen sie bei ihm die Abbildung einer anatomischen Lehrfigur, und sofort vermag sich Pécuchet für die nächste Disziplin zu begeistern: „Ce doit être une belle étude que l'anatomie ?« $(108)^{15}$ Und nicht nur in ihrem Wissensdrang, auch in ihrem Habitus und

12 „Sie] gerieten abermals in Bedrängnis beim Gesetz der Äquivalenzen. Sie bemühten sich, mit Hilfe der Atomtheorie Licht in die Angelegenheit zu bringen, aber da versagten sie vollends.« (65) »Indessen, all die Bücher hatten sie ganz wirr im Kopf gemacht.« (81) »Die Minerale ermüdeten sie bald [...].« (87)

13 Die Situationskomik dieser Szene ist unübersehbar: Pécuchet betont das "wissenschaftliche Ziel« ihres Tuns, und wie auf ein Stichwort geht die Geröllmasse nieder. Flaubert genügt dafür der lakonische Anschluss mit der Konjunktion alors: "C'est dans un but scientifique répondit Pécuchet. Alors une masse tomba, en les frôlant de si près tous les quatre, qu'un peu plus ils étaient morts." (138) - "Wir verfolgen ein wissenschaftliches Ziek, sagte Pécuchet. Mit einemmal fiel eine schwere Masse so dicht bei den vieren herab, daß sie um ein Haar verschüttet worden wären." (93)

14 Vgl. Hans Jost Frey, Flauberts Monotonie, in: Ders. (Hg.), Der unendliche Text, Frankfurt a.M. 1990, S. 202-229, zu Bouvard et Pécuchet S. 220-229.

15 "Die Anatomie ist gewiß ein interessantes Fach?» (66). 
ihren Praktiken zeigen sich Bouvard und Pécuchet als mustergültige Wissenschaftler des 19. Jahrhunderts. Immerhin, so Claudine Cohen, »errichten sie ein Labor für ihre chemischen Experimente, ein Museum zum Erhalt ihrer geologischen und archäologischen Funde und ein Netzwerk mit korrespondierenden Beratern ${ }^{16}{ }^{16}$

Die Frage ist also, wie sich die Haltung dieser beiden Romanfiguren zum Wissen näher beurteilen lässt. Vieles spricht dafür, Bouvard und Pécuchet im engeren Sinne des Wortes als Dilettanten zu bezeichnen, um ihre liebevolle Hinwendung zu allem irgendwie Interessierenden zu charakterisieren. Die Bezeichnung drängt sich gerade deswegen auf, weil der Dilettantismus in der professionalisierten und disziplinär differenzierten Wissenschaftskultur des 19. Jahrhunderts kaum noch als Option der Erkenntnisgewinnung in Betracht kommt. ${ }^{17}$ Die Flaubert'schen Helden sind aber nicht einfach nur historisch verspätete Amateure in Zeiten der 'Wissenschaft als Beruf. Vielmehr birgt ihr Dilettantismus eine Kritik der neuen, disziplinären Wissenschaftssystematik. ${ }^{18}$ Die Art und Weise, in der sie die Fachleute nachahmen, bewirkt eine Karikatur des Fachwissens, so wie es kapitelweise von Disziplin zu Disziplin abgeschritten wird; ihr Weg durch die Wissenswelten erscheint als Zerrbild wissenschaftlichen Fortschritts und entstellt dessen Ideologie zur Kenntlichkeit. Der Roman liest sich als Parodie des universitären Curriculums, und die stets unzureichende habituelle Einübung der Protagonisten in das Wissenschaftlerideal des 19. Jahrhunderts als Satire auf eben dieses Ideal und auf die damit einhergehende Wissenschaftsgläubigkeit der Moderne. ${ }^{19}$

16 Claudine Cohen, Bouvard et Pécuchet réécrivent les sciences, o.S.: "[Ils] mettent en place un laboratoire pour leurs expériences de chimie, un muséum pour conserver des spécimens de géologie et d'archéologie, organisent un réseau de correspondances avec des conseillers qui leur envoient livres, instruments et spécimens«. Vgl. Scholler, Umzug nach Encyclopaedia, mit einer ausführlichen Aufschlüsselung einzelner wissenschaftlicher Tätigkeiten der Protagonisten (z.B. Studieren, Dozieren, Experimentieren, Sammeln, Notieren).

$17 \mathrm{Zu}$ dieser Umgewichtung des Dilettantismus vgl. Stefan Blechschmidt/Andrea Heinz (Hg.), Dilettantismus um 1800, Heidelberg 2007.

18 Dahingehende Perspektivierungen des Dilettantismus im 19. und 20. Jahrhundert finden sich in: Safia Azzouni/Uwe Wirth (Hg.), Dilettantismus als Beruf, Berlin 2010. Zum Problem von Dilettantismus und Expertentum in Bouvard und Pécuchet vgl. Karin Westerwelle, Fiktionen des Wissens. Bouvard und Pécuchet, in: Merkur 66 (2012), Nr. 760, S. 971-979 (Doppelheft "Macht und Ohnmacht der Experten«).

19 Vgl. Claudine Cohen, Bouvard et Pécuchet réécrivent les sciences, o.S.: »Image inversée d'un siècle fasciné par le développement des sciences, l'itinéraire des deux employés est une critique décapante des savoirs positifs, une caricature de la foi naïve dans la science." 
Einschränkend ist jedoch zu sagen, dass Begriffe wie Satire, Parodie und Karikatur auf eine klare Wertung deuten, die Flauberts Roman gerade verweigert. Es fehlen wie auch immer geartete Hinweise darauf, wie ein anderer, sinnvollerer und womöglich nützlicherer Umgang mit Wissen denn aussehen könnte. Stattdessen beschränkt sich der Roman in seinem noch näher zu erörternden literarischen Verfahren auf das, was Roland Barthes die "Ausführung eines Textes« genannt hat - ein Verfahren, das, wie er ausdrücklich hinzufügt, "nicht durch die Parodie« zustande kommt, sondern durch die Kopie. Darin liegt die Bedeutung der (Berufs-)Tätigkeit der Protagonisten: "[D]ie beiden Kopisten sind Abschreiber von Codes«, nämlich jener »kulturellen Codes", die Barthes zufolge im realistischen Roman die "Vulgata des Wissens" (vulgate scientifique) bilden. ${ }^{20}$ Es ist daher von weitreichender Bedeutung, dass Bouvards und Pécuchets Weg durch das System der empirischen und schöngeistigen Wissenschaften schließlich - dem nicht zu Ende geführten Plan Flauberts zufolge - zurück zum alten Kopistentum führen sollte. Ihre fehlgehenden Versuche auf dem Feld des Wissens sollten also in die erprobte Praxis des Abschreibens münden.

Diese zirkuläre Struktur erzeugt im geplanten zwölften und letzten Kapitel des ersten Bandes eine mise en abyme. In Flauberts kurzem Entwurf finden Bouvard und Pécuchet einen Brief des kleinstädtischen Arztes, in dem sie als "zwei harmlose Schwachköpfe« bezeichnet werden (»deux imbéciles inoffensifs«). Dieser Brief sollte offenbar alle Handlungen und Gedanken der Helden nochmals zusammenfassen: "en résumant toutes leurs actions et pensées«. Der erste Teil des Romans sollte also mit einer ausgedehnten Zusammenfassung seiner selbst schließen, was zugleich eine Selbstkritik des Romans im Roman bedeutet hätte: "[la lettre] doit, pour le lecteur, être la critique du roman". Um das selbstreflexive Arrangement noch zu überbieten, sollten Bouvard und Pécuchet den Brief, der sie denunziert, schließlich unverdrossen abschreiben, wie alles andere auch, allerdings ausdrücklich unter Zurückweisung dieser Selbstreflexivität, »ohne Reflexion«: "Qu'allons-nous en faire ? - Pas de réflexion! copions ! « Damit wäre dann das Schlusstableau des ersten Bandes erreicht worden, das die beiden über ihre Pulte gebeugt beim Kopieren gezeigt hätte: „Finir par la vue des deux bonshommes penchés sur leur pupitre, et copiant.« (401)

20 Roland Barthes, S/Z (1970), übers. von Jürgen Hoch, Frankfurt a.M. 1987, S. 101. Die anderen Zitate lauten im französischen Original (in: Ders., Euvres complètes, hg. von Éric Marty, Bd. 2, Paris 1994, S. 555-741, hier S. 620): »en opérant un texte, non une parodie«; "C'est ce qu'a fait Flaubert dans Bouvard et Pécuchet : les deux copistes sont des copieurs de codes«. Vgl. dazu Baßler, Erhabene und komische Kopisten, S. 319. 
Liegt nicht in dieser Rückkehr zum Kopistentum sogar ein Erkenntnisfortschritt? Allerdings einer, der nicht mit dem swissenschaftlichen Fortschritt als solchem zu verwechseln wäre. Gerade diesem schwören Bouvard und Pécuchet ja schließlich ab, indem sie aus ihrem Scheitern die Konsequenz ziehen, sich nur mehr als Dokumentaristen und Archivare des Wissens zu betätigen. Damit werden sie letztlich selbst zu Enzyklopädisten, wie Flauberts Plänen für einen zweiten Band des Romans zu entnehmen ist.

\section{II.2 Textgenese: Wissen als Material des Romans}

Mit dem zweiten Band von Bouvard et Pécuchet hätte es eine merkwürdige Bewandtnis gehabt: Offenbar sollte er eben jene Abschriften enthalten, an die sich die Protagonisten zum Ende des ersten Bandes setzen (mit der zitierten Abschlussformel »ils s'y mettent«, 399), also eine fiktionsimmanente Sammlung von Kopien wissenschaftlicher Literatur. ${ }^{21}$ Was konkret vorliegt, sind aber die vom empirischen Autor Flaubert angefertigten Kopien, seine Notizen und Exzerpte. Demnach wäre der zweite Band insgesamt zur Dokumentation der Vorstudien des Romans geworden. In der Flaubert-Philologie wurde und wird intensiv diskutiert, welche unter den tausenden von nachgelassenen Blättern in ihrer "hétérogénéité profonde et polymorphe « ${ }^{22}$ als Grundlage des zweiten Bandes zu klassifizieren seien. Eine tatsächliche Textkonstitution bezeichnete schon Guy de Maupassant, der nach Flauberts Tod die erste Sichtung des Nachlasses übernommen hatte, als »unausführbar «, ${ }^{23}$ und fast alle Editoren sind ihm in dieser Einschätzung gefolgt. In den meisten Ausgaben beschränkt sich die Auswahl letztlich auf eine überschaubare Anzahl von Fragmenten, etwa die Notes des auteurs précédemment lus, der Catalogue des idées chic oder das Album de la marquise. ${ }^{24}$

21 Vgl. Jacques Neefs/Claude Mouchard, Vers le second volume: "Bouvard et Pécuchet«, in: Raymonde Debray-Genette (Hg.), Flaubert à l'œuvre, Paris 1980, S. 169-217; Anne Herschberg Pierrot, Ironie et érudition: le second volume de "Bouvard et Pécuchet«, in: Vincent Jouve/Alain Pagès (Hg.), Les Lieux du réalisme, Paris 2005, S. 257-283.

22 Stéphanie Dord-Crouslé, Fragments pour le second volume, in: Flaubert, Bouvard et Pécuchet, S. 403-409, hier S. 405.

23 Brief an Flauberts Nichte, 30.7.1881, zit. nach Stéphanie Dord-Crouslé, Fragments pour le second volume, S. 403: "je crois maintenant cette besogne inexécutable."

24 In der hier verwendeten Ausgabe finden sich - neben weiteren - die beiden erstgenannten Sammlungen. Die Gründe gegen eine Aufnahme des Album de la marquise nennt Stéphanie Dord-Crouslé, Fragments pour le second volume, S. 408f. Vgl. auch die umfangreiche deutsche Ausgabe: Gustave Flaubert, Universalenzyklopädie der menschlichen Dummheit, hg. und übers. von Hans-Horst Henschen, 2 Bde., Frankfurt a.M. 2004. 
Am bekanntesten unter diesen Fragmenten ist sicherlich der Dictionnaire des idées reçues. In dieser Kollektion von Gemeinplätzen interessieren sIdeen nicht in ihrem Gehalt, sondern in ihrem Charakter des Konventionellen, Herkömmlichen, Überkommenen (reçu); der Dictionnaire dokumentiert nicht eigentlich einen Wissensstand, sondern eine bestimmte Art des Bescheid wissenden Sprachgebrauchs. Dazu passt, dass die Einträge keiner sachlichen Systematik, sondern nur der Arbitrarität des Alphabets folgen. ${ }^{25}$ Mit dem Format des Wörterbuchs entsteht ein Gegengewicht zur curricularen Form des ersten Romanteils. Umso deutlicher wird, dass sich die vermeintliche Expertise der idées reçues unterschiedslos auf alles Mögliche richtet. Die Einträge lauten etwa: »ABSINTHE. Poison extra-violent. A tué plus de soldats que les Bédouins !«, »FIĖVRE. Prouve la force du sang. Est causée par les prunes«, "LUNE. Inspire la mélancolie. Est peut-être habitée ?« oder »PALMYRE. Reine d'Égypte ? - ou ruines? on ne sait pas. ${ }^{26}$

Es werden also stark verengte oder gänzlich unzureichende Definitionen mit bestimmten Konversations-Topoi verknüpft, die selbst noch die Geste des philosophischen Staunens und Wissen-Wollens zum Klischee werden lassen. Viele der Einträge haben einen gleichsam memorativen Gestus, so als müsse man sich die Gemeinplätze immer neu einprägen: "EUNUQUE. Fulminer contre les castrats de la chapelle Sixtine", "STUART (Marie). S'apitoyer sur son sort « oder "VACCINE. Ne fréquenter que les personnes vaccinées. ${ }^{27}$ Da dieser Sprachgebrauch unkommentiert dokumentiert wird, handelt es sich in gewisser Weise um einen dictionnaire critique nach dem Muster Pierre Bayles - wenn auch ohne Nachweis einzelner Belegstellen, eher als Extrakt oder Essenz eines Sprechens im Modus der idées reçues. Die Intention dieser Sammlung ist jedoch kaum auf die kritische Entlarvung eines solchen Sprechens zu reduzieren. Gerade als Vorhaben der fiktiven Kompilatoren Bouvard und Pécuchet müsste man es sich vielmehr radikal affirmativ vorstellen.

Damit richtet sich nun nochmals der Blick auf die textkonstitutive Funktion des Wissens in Bouvard et Pécuchet insgesamt. Denn Wissen - szientifisches, historisches, belletristisches sowie para- und pseudowissenschaftliches - ist nicht nur der Gegenstand von Bouvards und Pécuchets Bemühungen, sondern auch der Stoff, durch den sich Flaubert selbst hindurchgearbeitet hat.

25 Zum Zusammenhang mit der Konjunktur des Konversationslexikons im 19. Jahrhundert vgl. Andreas Kilcher, Mathesis und poiesis, S. $304 f$.

26 Diese und die folgenden Nachweise aus der zitierten Ausgabe, Gustave Flaubert, Bouvard et Pécuchet, S. 417-450, unter dem jeweiligen alphabetischen Eintrag.

27 Ebd. 
Schon lange vor Bouvard et Pécuchet hatte er die Technik entwickelt, vor der eigentlichen Niederschrift seiner Erzähltexte ausführliche Stoffsammlungen anzulegen: Nicht nur für die historisch-orientalistischen Szenarien von Salammbô und Hérodias, sondern auch für die zeitgenössisch-zeitgeschichtlichen von Madame Bovary oder L'éducation sentimentale betrieb er jahrelange Vorstudien. Wie kaum einem anderen Autor des 19. Jahrhunderts war Flaubert daran gelegen, den Gegenstandsbereich seiner Werke jenseits des bloßen Sujets in ein geradezu uferloses Forschungsfeld auszuweiten. sStoff< meint also sowohl das Thematische des Gegenstands als auch seine Materialität, und er deutet bei Flaubert vor allem auf den Stoffwechsel, auf denjenigen Umwandlungsprozess, in dem der Stoff zu literarischem Text wird.

Für seinen letzten Roman steigerte Flaubert die Arbeit am Material bis zum Exzess. ${ }^{28}$ Die Fülle der von ihm studierten Literatur aus verschiedenen Disziplinen ist geradezu legendär, wobei er in seinen begleitenden Selbstkommentaren an der Legendenbildung bereitwillig mitwirkte. Zu Beginn des letzten Arbeits- und Lebensjahres veranschlagte er die Anzahl der durchgearbeiteten Bände auf mehr als 1500 und die Dicke seiner Notizenmappe auf acht Daumen. ${ }^{29}$ Mit wachsender Quantität intensivierte sich die Mühe bei der Umwandlung dieses Stoffes, was sich in Flauberts Briefen in einer durchgängigen Metaphorik des Metabolismus ausdrückt. Immer wieder betont er Akte des Einverleibens (innerhalb eines Jahres habe er 194 Bände "verschlungen«, er sei mit Lektüre "gemästet ${ }^{30}$ ) und imaginiert hyperbolische Ausscheidungsvorgänge: Über seine Zeitgenossen will er »den Ekel ausspucken, den sie in mir erregen [...], meinen Groll ausdünsten, meinen Hass auskotzen, meine Galle auswürgen, meine Wut abspritzen, mich von

28 Vgl. Jacques Neefs, Couper, copier, coller. Les dossiers de "Bouvard et Pécuchet«, in: Sonya Stephens (Hg.), Esquisses/ébauches. Projects and Pre-Texts in Nineteenth-Century French Culture, New York u.a. 2007, S. 113-121; Atsushi Yamazaki, "Bouvard et Pécuchet" ou la pulvérisation de la philosophie, in: Études de langue et littérature françaises 90 (2007), S. 81-100. - Die Materialien befinden sich in der Bibliothèque municipale von Rouen und sind seit 2013 online zugänglich, vgl. http://flaubert.univ-rouen.fr/bouvard_et_pecuchet [Datum des letzten Zugriffs: 23.12.2015].

29 „Savez-vous à combien se montent les volumes qu'il m'a fallu absorber pour mes deux bonshommes ? À plus de 1500 ! Mon dossier de notes a huit pouces de hauteur (an Edma Roger de Genettes, 24./25.1.1880, Correspondance, Bd. 5, S. 796).

30 "Savez-vous combien j'ai avalé de volumes depuis le 20 septembre dernier ? 194 !« (an Edma Roger de Genettes, 4.8.1873, Correspondance, Bd. 4, S. 695); "Je suis gorgé de lectures pieuses« (an Iwan Turgenjew, 8.11.1879, Correspondance, Bd. 5, S. 737). 
meinem Ärger reinigen« $3^{31}$ bevor er »krepiert«, möchte er seinen Mitmenschen noch »ein paar Kübel Scheiße« über den Kopf kippen. ${ }^{32}$

Flaubert hat die Wissensverarbeitung aber nicht nur metabolistisch übersteigert, sondern führt sie auch in gleichsam skelettierter Weise vor. Denn in Bouvard et Pécuchet bleibt der Anteil an Angelesenem, Gesammeltem so deutlich erkennbar wie in keinem anderen seiner Texte. Immer wieder gibt es weite Passagen mit Exzerptcharakter, oft unter genauer Nennung der ausgewerteten Fachliteratur. Diese Passagen sind von programmatischer Tragweite: In ihnen wird nicht einfach nur korrekt das zugrunde liegende Material nachgewiesen, sondern die Lektüre- und Nachweistechnik selbst wird dokumentiert. Allerdings kommt dieses Dokumentarische - sofern der erste Teil des Romans fertig gestellt worden ist - nicht in roher, unbearbeiteter Form vor; vielmehr wird man gerade dort, wo das Material besonders deutlich zu Tage tritt, die intensivste Bearbeitung durch den manischen Stilisten Flaubert vermuten dürfen. ${ }^{33}$ Daraus resultiert der kennzeichnende Stil dieses Romans: eine eigentümliche Mischung aus Exzerpt und Figurenrede. Hier ein Beispiel aus den philosophischen Studien des achten Kapitels. Bouvard und Pécuchet entdecken soeben für sich den Skeptizismus:

Mais si l'idée est spirituelle, comment représenter la matière ? De là, sceptisicme quant aux perceptions externes. Si elle est materielle, les objets spirituels ne seraient pas représentés ? De là, sceptisicme en fait de notions internes. "D'ailleurs qu'on y prenne garde ! Cette hypothèse nous mènerait à l'athéisme !« Car une image étant une chose finie, il lui est impossible de représenter l'infini.

- »Cependant « objecta Bouvard »quand je songe à une forêt, à une personne, à un chien, je vois cette forêt, cette personne, ce chien. Donc les idées les représentent." Et ils abordèrent l'origine des idées.

31 »Tout cela dans l'unique but de cracher sur mes contemporains le dégoût qu'ils m'inspirent. Je vais enfin dire ma manière de penser, exhaler mon ressentiment, vomir ma haine, expectorer mon fiel, éjaculer ma colère, déterger mon indignation" (an Léonie Brainne, 5.10.1872, Correspondance, Bd. 4, S. 583).

32 "Et je ne voudrais pas crever avant d'avoir déversé encore quelques pots de merde sur la tête de mes semblables« (an Iwan Turgenjew, 8.11.1879, Correspondance, Bd. 5, S. 737).

33 Das Ausmaß der Bearbeitung lässt sich durch minutiösen Abgleich der Vorstudien mit dem Romantext im Einzelnen nachweisen. Eine solche mikrologische Auswertung der Exzerpte, am Beispiel von Flauberts Vorstudien zu Kapitel 5 (in dem sich Bouvard und Pécuchet als Literaten betätigen), findet sich etwa bei Stéphanie Dord-Crouslé, Le beau dans tous ses états. Le moment esthétique dans »Bouvard et Pécuchet«, in: Gisèle Séginger (Hg.), Gustave Flaubert 6: Fiction et philosophie, Caen 2008, S. 85-107. Dord-Crouslé sieht den Roman als "univers intertextuel complexe«, gekennzeichnet durch »le montage et la mise en fiction« der philosophischen Versatzstücke (ebd., S. 100). 
D'après Locke, il y en a deux, la sensation, la réflexion. Condillac réduit tout à la sensation. $(291)^{34}$

Auch die hier verhandelten `Ideen ‘ des schulmäßig philosophischen Diskurses sind vor allem als süberkommener, als idées reçues von Interesse. Das von den Protagonisten rekapitulierte philosophische Lehrbuchwissen, hier die säuberliche Dichotomie des Skeptizismus hinsichtlich der perceptions externes und der notions internes, ist im Indikativ Präsens notiert, wird also ohne ausdrückliche Signale der indirekten Rede gleichsam eins zu eins im Text abgebildet. Bouvard und Pécuchet ziehen aus dem Gelesenen Schlüsse, die sie für ihre eigenen halten, die aber dennoch bloße Gemeinplätze sind, wie die Warnung vor dem Atheismus, die in wörtlicher Rede als gegenseitiger oder zweistimmiger Zuruf erscheint (»D'ailleurs qu'on y prenne garde ! Cette hypothèse nous mènerait à l'athéisme !«), oder Bouvards Raisonnement über die Repräsentationsfunktion der Ideen. Die Erzählerfigur hält sich auf die für Flaubert typische Weise weitestgehend zurück, benennt nur kurz den Übergang zum nächsten Forschungsfeld und überlässt dann wieder das Feld den Protagonisten, ihren beschränkten Reflexionen und ihren Dialogen mit anderen, ähnlich beschränkt reflexionsfähigen Figuren.

Wie schwierig diese Struktur ist, merkt man daran, dass der Perspektivismus-Experte Flaubert bisweilen die Maskerade nicht durchhält, so als könne er es momentweise nicht ertragen, seine farcenhafte Kritik des Wissens nur durch die doppelte persona seiner Protagonisten zu äußern. Daraus resultieren kurze Anflüge von Auktorialität, also unvermittelte Einblendungen einer Erzählerfigur, die sich von den Hauptfiguren distanziert. Diese Stellen bewirken kurze Öffnungen im Zeitgefüge der Erzählung: Während normalerweise alles Geschehen durch die 'Reflektorfiguren Bouvard und Pécuchet vermittelt wird, also immer nur in ihrer jeweiligen Gegenwart stattfinden kann, ${ }^{35}$ ist dieser Erzähler zu ganz anders gearteten Aussagen imstande, etwa wenn es heißt, bestimmte wissenschaftliche Abhandlungen

34 »Wenn aber die Idee unkörperlich ist, wie läßt sich dann die Materie darstellen? Daher der Zweifel an den Wahrnehmungen von außen. Ist sie materieller Natur, wäre dann das Geistige nicht vorstellbar? Daher der Zweifel hinsichtlich der inneren Wahrnehmungen. Übrigens ist hier Vorsicht geboten! Diese Hypothese könnte zum Atheismus führen. ‘ Indes`, wandte Bouvard ein, ‘wenn ich an einen Wald, an einen Menschen, an einen Hund denke, sehe ich diesen Wald, diesen Menschen, diesen Hund. Folglich werden sie durch die Ideen dargestellt. $<$ Und sie wandten sich dem Ursprung der Ideen zu. Nach Locke gibt es deren zwei: die sinnliche Wahrnehmung und das Denken, und Condillac führt alles auf die sinnliche Wahrnehmung zurück.« (240)

35 Vgl. die Gegenüberstellung von Erzählerfigur und Reflektorfigur bei Franz K. Stanzel, Theorie des Erzählens, 4. Aufl., Göttingen 1989, S. 190-239. 
seien »zu jener Zeit« berühmt gewesen, oder wenn unvermittelt eine kurze Vorausdeutung über "viele Jahre" erfolgt. ${ }^{36}$

Auch wenn dieser Abstand zwischen dem Erzähler und dem perspektivischen Protagonisten-Doppel immer nur kurz aufscheint, markiert er doch eine für das Zeitmanagement des Romans entscheidende Distanz. Diese Distanz lässt sich quantifizieren: Das Schreiben des Notars, mit dem Bouvard im ersten Kapitel zur Testamentseröffnung geladen wird, ist auf den 14. Januar 1839 datiert. ${ }^{37}$ Die Handlung vollzieht sich also im Verlauf der 1840er-Jahre, was bedeutet, dass der in den 1870er-Jahren verfasste Roman durchweg drei Jahrzehnte zurückblickt. Das ist nicht nur ein erzählzeitliches, sondern auch ein wissenshistorisches Argument. Aus der Fortschrittslogik des 19. Jahrhunderts betrachtet, bewegen sich Flauberts Protagonisten durchgehend im Bereich des Veralteten, ja Obsoleten - am deutlichsten gewiss angesichts des Umstands, dass sie sich eine Wissenswelt vor Darwin erschließen, womit ihre Begriffsstutzigkeit im Bereich der Arten- und auch der Erdgeschichte in besonderer Weise pointiert erscheint. ${ }^{38} \mathrm{Im}$ Sinne der historischen Epistemologie entsteht jedoch gerade aus dieser Distanz eine Dialektik von sich fortwährend veränderndem Wissen und retrospektiv organisierter Wissens- und Wissenschaftsgeschichte. Ohne dass im oben erwähnten Verständnis einfach von Satire oder Parodie die Rede sein müsste, entsteht so ein iromisches Verhältnis zur Emphase des wissenschaftlichen Fortschritts.

,Wissen dringt also fortwährend in den Romantext ein und speist ihn unausgesetzt. Es ist auf seltsame Weise unnütz und nutzbringend zugleich. Was auf der Figuren- und Handlungsebene des Romans als ziel- und nutzlos klassifiziert werden kann, ist auf der Ebene der Textkonstitution immer schon nützlich, im Sinne des Produktiven, des Funktionalen, der Wirksamkeit. So gesehen steht Bouvard et Pécuchet zweifellos in der wissenspoetologischen Kontinuität des französischen Romans, der im 19. Jahrhundert die Wissenschaften »reflektiert, anwendet und umschreibt«, um so - weit jenseits vermeintlich realistischer Nachahmungspostulate - die »Authentizität

36 »...] un bouquiniste leur procura les traités de Richerand et d'Adlon, célèbres à l'époque« (113; dt. 70); "et pendant plusieurs années, ils se détournaient dans la campagne, sitôt qu'apparaissait un chien, ressemblant à celui-là巛 (117; dt. 74: es geht um Bouvards und Pécuchets noch Jahre währende Furcht vor einem Hund, mit dem sie experimentiert haben).

37 Vgl. 57 (dt. 16).

38 Vgl. Anthony Zielonka, Fossils and Theories of Evolution in Gustave Flaubert's »Bouvard et Pécuchet«, in: Flaubert. Revue critique et génétique 5 (2011), http://flaubert.revues.org/1337?lang=en [Datum des letzten Zugriffs: 23.12.2015]. 
und Bedeutsamkeit der Erzählung zu beglaubigen «. ${ }^{39}$ Auch wenn man den Stellenwert des Umschreibens bei Flaubert wesentlich stärker gewichten muss als den der Beglaubigung, bleibt doch der Nutzen des von ihm angeeigneten Wissens in funktioneller Hinsicht unübersehbar. Bouvard et Pécuchet ist auch das Ergebnis angewandter Wissenschaft.

\section{II.3 Poetologie: Flauberts Romankunst als das Andere des Wissens}

Bei aller Produktivität der Wissensverarbeitung: Flauberts briefliche Äußerungen aus der Entstehungszeit von Bouvard et Pécuchet zeugen weniger vom Stolz des Wissenserwerbs als vielmehr von einer für die moderne Literatur exemplarischen Selbstpathetisierung. Die leidvolle Arbeit dieses enzyklopädischen Autors bestand darin, immer mehr Wissen zu schlucken, bis zur Erstickung, weil er (in Umkehrung des berühmten Max-Liebermann-Zitats) nicht soviel kotzen konnte, wie er fressen musste. Auch wenn er Bouvards und Pécuchets Pathos des Opfers für die Wissenschaft deutlich ironisch stilisierte, ${ }^{40}$ pathetisierte er doch rückhaltlos sein eigenes Leiden am Wissen, das er auf sich nahm, um sein letztes Werk als Monument der modernen Kunst zu errichten. Denn das in seiner Vielfalt ungestalte Material war in einen stilistisch bis ins letzte Detail durchgearbeiteten literarischen Text umzuwandeln, der aber trotzdem die ihm zugrunde liegende Heterogenität und Heteronomie deutlich, ja überdeutlich ausstellen sollte. Es war dieser mehrfache Widerspruch zwischen der textkonstitutiven und der poetologischen Funktion von Wissen, an dem Flaubert, emphatisch gesprochen, schließlich zugrunde ging, als er in der Arbeit am Ende des ersten Bandes seine finale Hirnblutung erlitt.

Der Weg in die Affirmation des Wissens, den vor ihm Balzac beschritten hatte und den Zola seit den 1860er-Jahren mit Begeisterung verfolgte, war für Flaubert also ausgeschlossen. Dennoch wird sein Stil oft mit Wissenschaftlichkeit assoziiert, vor allem mit szientifischer Objektivität und Kühle. Auch viele seiner Selbstdeutungen zielen in diese Richtung. Schon früh deklarierte er: »La littérature prendra de plus en plus les allures de la scien-

39 Claudine Cohen, Bouvard et Pécuchet réécrivent les sciences, o.S.: „Le roman du XIXe siècle réfléchit, applique, réécrit les sciences. [...] [T] out se passe comme si la caution de la science apparaissait alors nécessaire pour valider l'authenticité et la pertinence du récit romanesque."

40 »Ils ambitionnaient de souffrir pour la science« (111), übersetzt als: »ihr Bestreben war es, für die Wissenschaft Opfer zu bringen« (68). 
ce ${ }^{41}$ verlangte also vom Schriftsteller, so Claudine Cohen, »dieselbe Objektivität, dieselbe Strenge und dieselbe Askese wie vom Wissenschaftler in seinem Laboratorium «. ${ }^{42}$ Doch selbst wenn die Literatur die allures de la science übernimmt, also wissenschaftliche Gangart und Geschwindigkeit, wird sie darum doch nicht mit der Wissenschaft identisch. Sonst wäre Bouvard et Pécuchet nicht nur von seinem Gegenstand /Wissenschaft‘, sondern auch von wissenschaftlicher Methodik nicht zu unterscheiden - was aber beides sehr wohl der Fall ist. Die nun folgenden, abschließenden Überlegungen gehen daher von der Hypothese aus, dass in Flauberts Poetologie das Wissen einen programmatisch anderen Stellenwert hat als das Schreiben. Der Roman als Ergebnis einer enormen Anstrengung des literarischen Schreibens ist das Andere des Wissens. Das heißt: Um zu schreiben, muss man aufhören zu wissen. Um aber mit dem Wissen aufhören zu können, muss man es sich zunächst einmal auf umfassendste Weise angeeignet haben. Um noch dazu einen Roman wie Bouvard et Pécuchet zu schreiben, muss man das angeeignete Wissen im Zuge des Schreibens auch noch explizit wie implizit zurückweisen.

Daraus resultiert die maximal anspruchsvolle Aufgabe, den Roman über das so beschränkte und doch so wissbegierige Figuren-Doppel Bouvard und Pécuchet zu perspektivieren. Angesichts dieser poetologischen Herausforderung relativiert sich Flauberts fortwährend bekundete Absicht, mit dem Roman eine Attacke auf die Dummheit seiner Epoche zu liefern. Das zeigt sich nicht zuletzt an der einzigen Stelle des Romans, an der Bouvard und Pécuchet selbst zu Kritikern der Dummheit werden, nachdem sie ihre philosophisch-kritischen Studien so weit getrieben haben, dass sie die Grundlagen der kleinstädtischen Gesellschaft von Chavignolles in Zweifel ziehen: "Alors une faculté pitoyable se développa dans leur esprit, celle de voir la bêtise et de ne plus la tolérer.« $(305)^{43}$ In dieser Passage des achten Kapitels erwägen die beiden den Suizid und bringen es bis zum Aufbau einer Vorrichtung zum Erhängen, scheitern aber geradezu slapstickhaft an der Ausführung. Daraufhin besinnen sie sich eines Besseren und machen sich auf den Weg zur Kirche. So entdecken sie ein neues Betätigungsfeld - die Religion in

41 Brief an Louise Colet, 6.4.1853, Correspondance, Bd. 2, S. 298.

42 Claudine Cohen, Bouvard et Pécuchet réécrivent les sciences, o.S.: "La littérature prendra de plus en plus les allures de la sciences, déclare Flaubert, demandant à l'écrivain la même objectivité, la même rigueur et la même ascèse que celles du savant dans son laboratoire."

43 »Da entwickelte sich in ihrem Verstand eine bedauernswerte Fähigkeit, nämlich die, die Dummheit zu sehen und sie nicht mehr dulden zu können.« (254) 
Theorie und Praxis - und finden zugleich ihren alten Antrieb wieder: »Une curiosité les y poussa.« $(309)^{44}$

Bouvard und Pécuchet kehren also von ihrer Kritik der Dummheit alsbald zu ihrer Suche nach Wissen zurück - einer Suche, bei der sie immerfort und immer weiter auf dem Weg der Dummheit bleiben. Diese Beharrungskraft der Dummheit ist in der französischen Literaturkritik um 1970 wiederholt als Charakteristikum des Romans hervorgehoben worden. Roland Barthes betonte in seiner bereits zitierten Reflexion über Bouvard und Pécuchet, dass sie in ihrer Funktion als Kopisten "dumm " (bêtes) seien. ${ }^{45}$ Gilles Deleuze notierte in einer Anmerkung zu Différence et répétition, Flaubert werfe Dummheit "auf philosophische Weise« auf, "als transzendentales Problem der Beziehungen zwischen Dummheit und Denken« ${ }^{46}$ Und Michel Foucault befand in seinem Nachwort zu Flauberts Tentation de Saint-Antoine, dass Bouvard et Pécuchet auf die Dummheit hinauslaufe, genauer auf die "Blödheit und Tugend, Heiligkeit und Dummheit derer, [...] die sich mit ihrer ganzen Existenz still abmühen, in verblendeter Verbissenheit wieder ihrer Natur innezuwerden. ${ }^{47}$ Neuerdings hat sich die Flaubert-Forschung in dem von Anne Herschberg Pierrot herausgegebenen Band Flaubert, l'empire de la bêtise (2012) dem Faszinosum der Dummheit wieder zugewendet. Dort wird unter anderem bemerkt, wie sehr Flaubert schon zu Beginn seiner Autorschaft von der Beschäftigung mit Dummheit besessen war; erst im Spätwerk jedoch sei sie ihm als bêtise mit großem B erschienen: "la Bêtise est devenue majuscule«, womit sie die Dimensionen des 'großen Tiers« (Bête) der Apokalypse gewonnen habe. ${ }^{48}$

44 »Neugierig machten sie sich auf den Weg dorthin." (259)

45 Roland Barthes, S/Z, S. 101, frz. S. 620.

46 Gilles Deleuze, Differenz und Wiederholung (1968), übers. von Joseph Vogl, München 1992, S. 344, Anm. 5. Im Original (Différence et répétition, Paris 1968, S. 353, Anm. 1): "comme problème transcendental des rapports de la bêtise et de la pensée«.

47 Michel Foucault, Nachwort [Un "fantastique« de bibliotheque, 1967], übers. von Hans Dieter Gondek, in: Michel Foucault, Schriften zur Literatur, hg. von Daniel Défert/ François Ewald, Auswahl von Martin Stingelin, Frankfurt a.M. 2003, S. 117-141, hier S. 139f. Im Original (Dits et écrits 1954-1988, hg. von Daniel Défert/François Ewald, Bd. 1, Paris 1994, S. 293-312, hier S. 311): "stupidité et vertu, sainteté et bêtise de ceux [...] qui s'efforcent silencieusement, toute leur existence, de rejoindre leur nature par un acharnement aveugle."

48 Anne Herschberg Pierrot, Avant-propos, in: Dies. (Hg.), Flaubert, l'empire de la bêtise, Nantes 2012, S. 7-14, hier S. 8. Als Beleg zitiert Herschberg Pierrot Flauberts briefliche Äußerung: "Voilà la vraie immoralité: l'ignorance et la Bêtise ! Le Diable n'est pas autre chose " (Brief an die Nichte Caroline, 12.1.1877, Correspondance, Bd. 5, S. 169). Vgl. dazu auch Pierre-Marc de Biasi, "Le Diable n'est pas autre chose", in: Anne Herschberg Pierrot (Hg.), Flaubert, l'empire de la bêtise, S. 35-67. 
Es geht hier also um eine weit mehr als kleinbürgerliche Dummheit, an der daher die bloß zeitkritische Satire scheitern muss. Nicht im Besserwissen, sondern erst in der komplexen literarischen Aufgabe des Wissens - im Doppelsinn von Arbeit und Verzicht - wird die Dummheit in ihrem vollen Umfang und ihrer wissenspoetologischen Faszination erkennbar. Somit kann Flauberts letzter Roman geradezu als eine Studie über die Verdummung, die Verblödung, die Idiotie verstanden werden. Das haben, im Anschluss an Jean-Yves Jouannais' Buch L'idiotie, ${ }^{49}$ Anne Herschberg Pierrot und Jacques Neefs betont: Bouvard und Pécuchet seien Idioten im Wortsinn des griechischen idiótes, 'Einzel-, Privatperson', dies allerdings in der burlesken - farcenhaften - Erscheinungsform des komischen Duos, das sich von der bürgerlichen Welt der Provinz unterscheidet und absetzt. ${ }^{50}$ Flaubert selbst nannte seine Protagonisten in einem Brief an Turgenjew "mes deux idiots «, 51 bezog die Kennzeichnung aber auch auf die Arbeit am Roman selbst: Man müsse verrückt sein, um solche Arbeiten in Angriff zu nehmen; das entstehende Werk sei möglicherweise idiotisch, werde aber jedenfalls nicht banal ausfallen. ${ }^{52}$ Der Roman handelt also nicht einfach von zwei Idioten - in der mehr oder weniger achtungsvollen Bedeutung des Wortes -, sondern ist selbst ein Projekt der Idiotie, ein idiotisches Projekt.

49 Jean-Yves Jouannais, Lidiotie, Paris 2003, dort S. 48 über Bouvard et Pécuchet.

50 Anne Herschberg Pierrot/Jacques Neefs, "Bouvard et Pécuchet« et la Bêtise, in: Anne Herschberg Pierrot (Hg.), Flaubert, l'empire de la bêtise, S. 127-166, hier S. 132. Ähnliches hat auch Botho Strauß in seinem Buch über die Idiotie beschrieben: »Verblödung als rebellischer Untergrund des Geistes interessierte Swift wie Flaubert. Ersterer erreichte sie selbst. Für Flaubert wurde sie die intime Partnerin seiner Wissensexzesse.« Botho Strauß, Lichter des Toren. Der Idiot und seine Zeit, München 2013, S. 6.

51 Brief an Iwan Turgenjew, 27.7.1877, Correspondance, Bd. 5, S. 265.

52 Vgl. Brief an Iwan Turgenjew, 19.7.1877, Correspondance, Bd. 5, S. 261: "Il faut être fou pour entreprendre des œuvres pareilles ! Celle-là, d'ailleurs, pourrait bien être idiote ? En tout cas, elle ne sera pas banale." 